\title{
Additive Effects of Genetic Variation in Dopamine Regulating Genes on Working Memory Cortical Activity in Human Brain
}

\author{
Alessandro Bertolino, ${ }^{1,3,4}$ Giuseppe Blasi, ${ }^{1,3}$ Valeria Latorre, ${ }^{1}$ Valeria Rubino, ${ }^{1}$ Antonio Rampino, ${ }^{1}$ Lorenzo Sinibaldi, ${ }^{2,6}$ \\ Grazia Caforio, ${ }^{1}$ Vittoria Petruzzella, ${ }^{2}$ Antonio Pizzuti, ${ }^{5,6}$ Tommaso Scarabino, ${ }^{4}$ Marcello Nardini, ${ }^{1}$ \\ Daniel R. Weinberger, ${ }^{3}$ and Bruno Dallapiccola ${ }^{5,6}$ \\ ${ }^{1}$ Psychiatric Neuroscience Group, Section on Mental Disorders, Department of Neurological and Psychiatric Sciences, and ${ }^{2}$ Department of Medical \\ Biochemistry and Medical Biology, University of Bari, 70124 Bari, Italy, ${ }^{3} \mathrm{Genes}$, Cognition, and Psychosis Program, National Institute of Mental Health, \\ National Institutes of Health, Bethesda, Maryland 20892, ${ }^{4}$ Department of Neuroradiology, Istituto di Ricovero e Cura e Carrattere Scientifico "Casa Sollievo \\ della Sofferenza," 71013 San Giovanni Rotondo (Foggia), Italy, ${ }^{5}$ IRCCS-CSS Mendel, 00198 Rome, Italy, and ${ }^{6}$ Department of Experimental Medicine and \\ Pathology, University La Sapienza, 00100 Rome, Italy
}

Functional polymorphisms in the catechol-O-methyltransferase (COMT) and the dopamine transporter (DAT) genes modulate dopamine inactivation, which is crucial for determining neuronal signal-to-noise ratios in prefrontal cortex during working memory. We show that the COMT Met ${ }^{158}$ allele and the DAT $3^{\prime}$ variable number of tandem repeat 10-repeat allele are independently associated in healthy humans with more focused neuronal activity (as measured with blood oxygen level-dependent functional magnetic resonance imaging) in the working memory cortical network, including the prefrontal cortex. Moreover, subjects homozygous for the COMT Met allele and the DAT 10-repeat allele have the most focused response, whereas the COMT Val and the DAT 9-repeat alleles have the least. These results demonstrate additive genetic effects of genes regulating dopamine signaling on specific neuronal networks subserving working memory.

Key words: dorsolateral prefrontal cortex; anterior cingulate; working memory; dopamine; catechol- 0 -methyltransferase; dopamine transporter

\section{Introduction}

Dopaminergic modulation of neuronal signal-to-noise is crucial for working memory processes in prefrontal cortex. More specifically, dopamine directly regulates firing of pyramidal neurons and of their GABA inhibitory surround within prefrontal cortex by differentially acting on $\mathrm{D}_{1}$ and $\mathrm{D}_{2}$ dopamine receptors in these neurons to focus prefrontal cortical resources to the task at hand (Seamans and Yang, 2004). Moreover, dopamine indirectly modulates prefrontal signal-to-noise via effects in the striatum, which regulates activity within the cortico-striato-thalamo-cortical circuit (Newman and Grace, 1999). A critical step in determining dopamine signaling is its removal from the extracellular space. Different mechanisms account for inactivation of dopamine at the synaptic and extrasynaptic levels, including methylation by catechol-O-methyltransferase (COMT) and reuptake via the dopamine transporter (DAT). COMT is expressed in neurons in the prefrontal cortex (Karoum et al., 1994), especially at neuronal dendritic processes in large pyramidal neurons (Matsumoto et

Received Nov. 22, 2005; revised Jan. 30, 2006; accepted Feb. $27,2006$.

We acknowledge Riccarda Lomuscio, Dr. Mariapia De Candia, Dr. Savino Dimalta, and Dr. Leonardo Fazio for help with data acquisition and all subjects who participated to the study.

Correspondence should be addressed to Dr. Alessandro Bertolino, Dipartimento di Scienze Neurologiche e Psichiatriche, Universita' degli Studi di Bari, Piazza Giulio Cesare, 9, 70124 Bari, Italy. E-mail: bertolia@psichiat.uniba.it. DOI:10.1523/JNEUROSCI.4975-05.2006

Copyright $\odot 2006$ Society for Neuroscience $\quad$ 0270-6474/06/263918-05\$15.00/0 al., 2003), with a distribution similar to that of $\mathrm{D}_{1}$ receptors (Diop et al., 1988). Moreover, both genetic and pharmacologic variations in COMT activity modify dopamine levels in the prefrontal cortex (Huotari et al., 2002; Tunbridge et al., 2004). The COMT gene contains a $\mathrm{G}$ to A missense variant (Lachman et al., 1996) that translates into a substitution of methionine for valine at codon 158 ( $\mathrm{Val}^{158} \mathrm{Met}$ ), such that the enzyme containing Met ${ }^{158}$ has significantly less activity and presumably greater synaptic dopamine than the $\mathrm{Val}^{158}$ enzyme (Lotta et al., 1995; Chen et al., 2004). Consistently, human subjects carrying the high-activity Val allele show greater engagement of cortical resources during working memory [as assessed with blood oxygen level-dependent (BOLD) functional magnetic resonance imaging (fMRI)] along with lower behavioral performance (Egan et al., 2001; Mattay et al., 2003; Bertolino et al., 2004b; Blasi et al., 2005).

The DAT critically regulates duration of the cellular actions of dopamine and the extent to which dopamine diffuses in the extracellular space, especially in the striatum. Expression of the DAT is abundant in the striatum where it is mostly found in synapses (Sesack et al., 1998; Lewis et al., 2001). In contrast, in the primary sensory motor cortex, and in prefrontal and cingulate cortices, the DAT is found in low abundance and primarily at a distance from synaptic sites of dopamine release (Sesack et al., 1998; Lewis et al., 2001). Given its ultrastructural localization, the DAT in the striatum is critical in regulating point-to-point dopa- 
mine neurotransmission, whereas in the cortex, the DAT seems to be better situated to regulate dopamine volume transmission (the three-dimensional spillover of dopamine from a release site reaching the extrasynaptic space) (Cragg and Rice, 2004). Volume transmission is thought to mediate interneuronal communication along with synaptic point-to-point transmission (Zoli et al., 1998). Considering its anatomical distribution and the importance in dopamine neurotransmission, the DAT may play a critical role in regulating cortical signal-to-noise ratio during working memory both directly and indirectly. DATs can directly affect prefrontal pyramidal neurons by regulating dopamine volume transmission on surrounding GABA inhibitory neurons. In contrast, the effect of the DAT can also be indirect via the corticostriato-thalamo-cortical pathway in which the net effect of dopamine in the striatum is of increasing activity of thalamo-cortical pathways with facilitation of cortically initiated action (Newman and Grace, 1999; Mattay et al., 2002). A functional variable number of tandem repeat (VNTR) polymorphism in the $3^{\prime}$ untranslated region of the DAT gene has been described previously (Vandenbergh et al., 1992). Alleles of this polymorphism range from 3 to 11 repeats, with the 9 - and 10-repeat alleles by far the most common (Vandenbergh et al., 1992). Compared with the 9-repeat allele, the 10-repeat allele has been associated with increased gene expression both in vitro (Mill et al., 2002) and in vivo (Heinz et al., 2000), although reports of the opposite allelic associations have also appeared (van Dyck et al., 2005). A more recent study under carefully controlled experimental conditions using the DAT protein itself as the reporter signal reported data consistent with the 10-repeat allele being associated with greater expression (VanNess et al., 2005). This study used a targeted stable integration protocol that eliminates confounds to construct comparison attributable to variable transfection efficiency or clonal variance, both common limitations of transient transfection or conventional nontargeted stable integration approaches. These limitations have been generally present in previous studies that have also used heterologous DAT.

Although the potential influence of additive genetic variation on complex behavioral phenotypes has long been predicted, there is no in vivo demonstration in humans of the effect of two genes acting together on the same neurobiology. We used fMRI in healthy subjects to explore the relationship of these two functional polymorphisms in dopamine-related genes and neuronal activity measured during working memory, known to involve prefrontal and cingulate activity. Based on the physiological role of dopamine in regulating signal-to-noise ratios in prefrontal neurons (both directly and indirectly via cortico-striatothalamo-cortical pathways), we hypothesized that individuals homozygous for the COMT Met allele and the DAT 10-repeat allele would show the most focused cortical engagement during working memory compared with COMT Val and DAT 9-repeat.

\section{Materials and Methods}

Subjects. We studied 62 healthy subjects (33 males; mean age, $31.8 \pm 8.1$ ). After genotype determination, the groups were divided based on COMT, DAT, and COMT-DAT genotypes. The $n$ values were as follows: COMT (Val/Val, 14; Val/Met, 34; Met/Met, 14), DAT (9/9-repeat, 7; 9/10repeat, 30; 10/10-repeat, 25), COMT-DAT (Val/Val 9/10-repeat, 6; Val/ Val 10/10-repeat, 8; Val/Met 9/9-repeat, 4; Val/Met 9/10-repeat, 17; Val/ Met 10/10-repeat, 13; Met/Met 9/9-repeat, 3; Met/Met 9/10-repeat, 7; Met/Met 10/10-repeat, 4). There were no subjects homozygous for COMT val and DAT 9-repeat alleles in this sample. The allelic distribution of both genes was in Hardy Weinberg equilibrium (COMT df 2, $\chi^{2}=0.58, p>0.2$; DAT df $\left.2, \chi^{2}=0.2, p>0.1\right)$. For additional demo- graphics and genotype determination, see supplemental material (available at www.jneurosci.org).

Working memory paradigm. During fMRI, all subjects completed a blocked paradigm of the N-Back task (Bertolino et al., 2004b), which includes a nonmemory guided control condition (0-Back) and a working memory condition (2-Back). Behavioral performance was recorded as accuracy and reaction time (see supplemental material, available at www.jneurosci.org).

Acquisition and analysis of imaging data. See supplemental material (available at www.jneurosci.org). BOLD fMRI data were acquired with a General Electric (Milwaukee, WI) 3T scanner, as described previously (Bertolino et al., 2004a,b). Data processing with SPM99 included reconstruction, registration, linear detrending, global normalization, and smoothing (10 mm Gaussian kernel). Individual contrast maps were created with $t$ statistics (2-Back $>0$-Back) and then used in second-level random effects models. All statistical analyses were thresholded at $p<$ 0.005 , minimum cluster size $(k)=3$, with additional Family Wise Error (FWE) small volume correction for multiple comparisons $(p<0.05)$. To identify the independent contribution of both genotypes to activation of the working memory cortical network, we performed multiple regression analyses within SPM99. For this analysis, we entered the single-subject contrasts (2-Back $>0$-Back) with the number of Met alleles of COMT genotype (Val/Val, 0; Val/Met, 1; Met/Met, 2) and with the numbers of 10-repeat alleles of DAT genotype (9/9-repeat, $0 ; 9 / 10$-repeat, $1 ; 10 / 10$ repeat, 2) as predictors. Furthermore, to evaluate the effect of both genotypes, we used linear regression analyses within SPM99 entering the single-subject contrasts with Met- and 10-repeat alleles as predictors (Val/Val 9/10-repeat, 0; Val/Val 10/10-repeat, 1; Val/Met 9/9-repeat, 2; Val/Met 9/10-repeat, 3; Val/Met 10/10-repeat, 4; Met/Met 9/9-repeat, 5; Met/Met 9/10-repeat, 6; Met/Met 10/10-repeat, 7). We used this analysis to evaluate the parametric effect of both genes with COMT genotype as the major grouping factor for a series of reasons. First, the effect of the COMT polymorphism was already known. Second, the presumed neurobiology of cortical and subcortical dopamine inactivation inside and outside of synapses (see above) makes it reasonable to hypothesize a greater effect of COMT versus that of DAT on cortical signal-to-noise. Third, we calculated the effect size of both genes and found that it was greater for COMT than for DAT. For anatomical localization, statistical maxima of activation were converted to conform to the standard space of Talairach and Tournoux. Brodmann areas (BAs) were determined using the Talairach Daemon software (http://ric.uthscsa.edu/projects/ talairachdaemon.html). To determine the proportion of variance in BOLD signal accounted for by both genotypes, we used $\omega^{2}$ (see supplemental material, available at www.jneurosci.org).

\section{Results}

Demographics and working memory performance

All genotype groups were matched for age, gender, full scale IQ, and handedness (all, $F<1.1$; all, $p>0.3$ ). They also did not differ in behavioral performance of the working memory tasks (accuracy and reaction time; all, $F<1.5$; all, $p>0.15$ ), thus allowing us

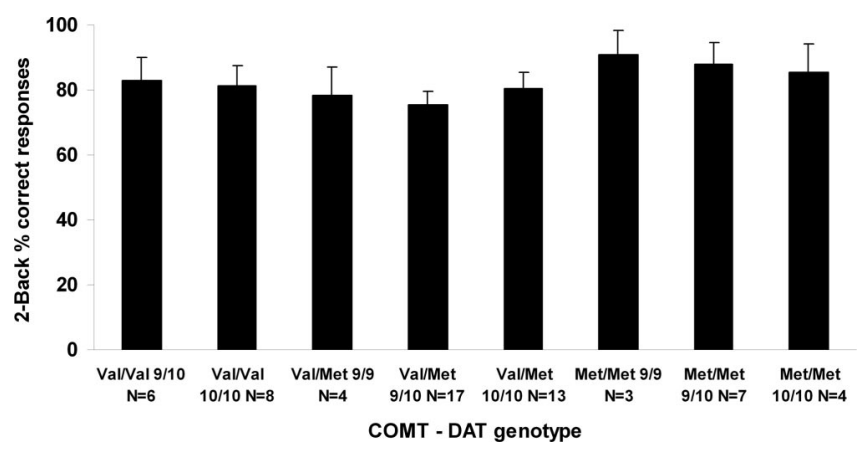

Figure 1. Working memory behavioral results. Performance accuracy results for the 2-Back task across genotype groups. Performance is represented as mean percentage of correct responses ( \pm SEM). 
to examine the effect of complex genotypes on brain activity independent of behavioral variation in this sample (Fig. 1). All of these analyses were performed separately for each genotype as well as for both genotypes together.

\section{Imaging data}

Analysis of the working memory imaging data in the whole sample revealed significant BOLD responses in the working memory cortical and subcortical network, including dorsolateral prefrontal cortex (BA 9), anterior cingulate (BA 24 and BA 32), premotor area (BA 6), parietal cortex (BA 39/40), caudate, and putamen, consistent with previous reports (Callicott et al., 1999, 2000; Egan et al., 2001; Bertolino et al., 2004b). Moreover, multiple regression analyses revealed that both genotypes independently predicted responses in areas of the working memory network. More specifically, the number of Met alleles in the COMT gene was negatively associated with response in the left precentral gyrus/left middle frontal gyrus (Brodmann areas 6/9; Talairach coordinates $x-40, y 1, z 27, k=27, t=3.37 ; p=$ 0.02 after FWE correction for multiple comparisons), in the right middle frontal gyrus (BA 9; $x 33, y 5, z 22, k=8, t=3.28$; $p=0.02)$, and in the anterior cingulate (BA 24; $x 11, y 12, z 27, k=26, t=3.37$; $p=0.02$ ) (Fig. 2, first row), again consistent with previous reports. Similarly, the number of 10-repeat alleles in the DAT gene was negatively associated with response in the left middle frontal gyrus (Brodmann area 9, $x-19, y 26, z 26, k=3, t=3.5$, $p=0.01$; BA $8, x-51, y 9, z 38, k=3, t=3, p=0.04)$, in the right middle frontal gyrus (BA 9/8; $x 33, y 16, z 32, k=25, t=3.61 ; p=$ 0.01 ), and in the anterior cingulate (BA 24; $x 8, y 12, z 27, k=3$, $\mathrm{t}=2.89 ; p=0.05$ ) (Fig. 2, second row). This approach allows for the unbiased determination of the contribution of independent variables to response of the working memory cortical network, suggesting that both genotypes are independently associated with it. We then used simple regression analyses with genotypes as regressors to address the effect of both genotypes together. Each genotype was attributed a different weight with COMT Val/ValDAT 9/10-repeat (there was no COMT Val/Val DAT 9/9 individual in this sample) the lowest weight and COMT Met/Met-DAT 10/10-repeat the highest. The results of this analysis indicated that subjects carrying Met and 10-repeat alleles have more focused engagement of areas BA 6/9 $(x-40, y 1, z 27, k=53, t=$ $3.44, p=0.01 ; x 33, y 5, z 22, k=10, t=3.36, p=0.02)$ and BA $24(x 11, y 12, z 27, k=29, t=3.44 ; p=0.01)$ than subjects carrying Val COMT and 9/10-repeat DAT (Fig. 2, third row), despite no differences in behavioral performance of the groups. This analysis indicates an overall effect on all groups. An additional $t$ test performed outside of image space indicated that the difference in percentage BOLD signal change between $\mathrm{Val} / \mathrm{Val}$ 9/10-repeat and Met/Met 10/10-repeat groups is statistically significant in all three clusters identified in the linear regression
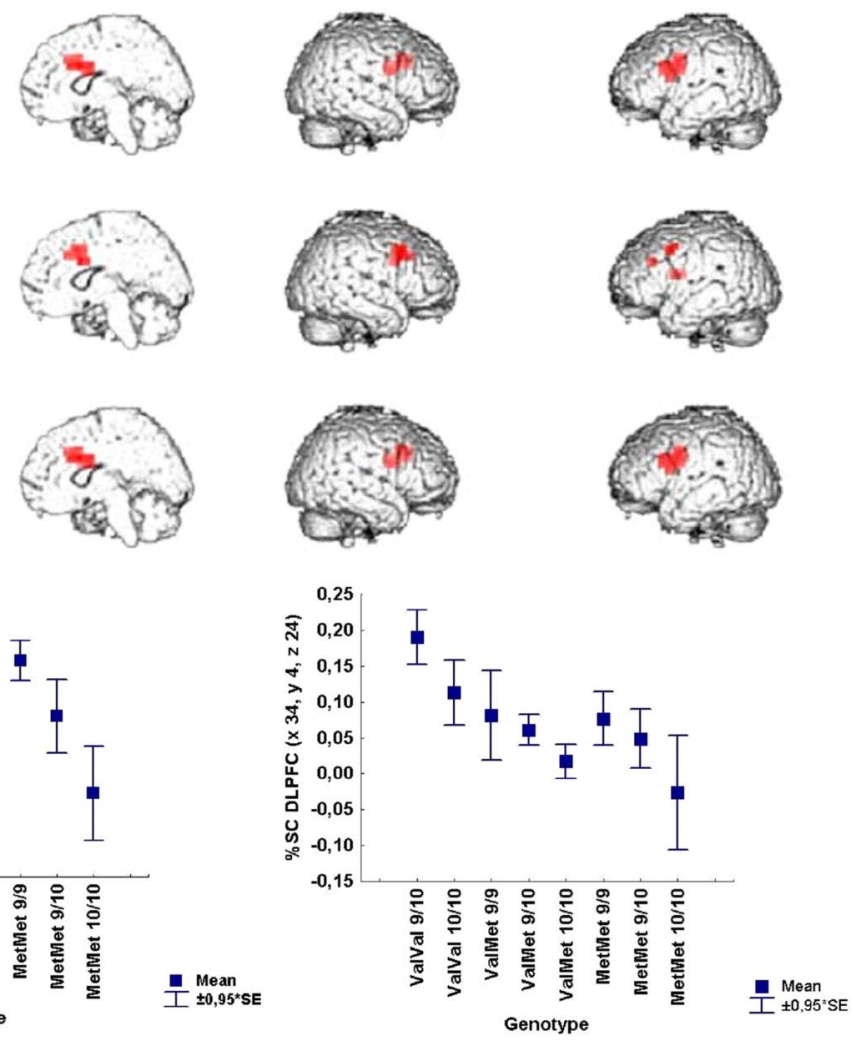

Genotype

Figure 2. Genotype-based analyses of the working memory BOLD fMRI response. First row, The effect of COMT with Met homozygous subjects having the most focused engagement of areas in BA 6, 9, and 24. Second row, The effect of DAT with 10-repeat homozygous subjects having the most focused engagement of areas in BA 9, 8, and 24. Third row, The combined effect 作 from the latter analyses in all eight genotype groups. These graphs suggest the additive effect of the two genotypes on percentage (df $=8$; all, $t>2.6$; all, $p<0.03$ ), suggesting that these two groups have the least and the most focused engagement.

The proportion of variance in the BOLD signal from left BA $6 / 9$, right BA 9, and BA 24 accounted for by both genotypes combined was 22,11 , and $16 \%$, respectively, as calculated with $\omega^{2}$. To further demonstrate the additive nature of the action of these two polymorphisms, we reasoned that by inverting the sign of the DAT polymorphism, we would dilute the COMT effect. Therefore, we performed another linear regression with COMT Val/Val-DAT 10/10-repeat having the lowest weight and COMT Met/Met-DAT 9/9-repeat having the highest. This analysis dramatically changed the results, because there was no negative relationship between genotypes and BOLD activation even dropping the statistical threshold at $p<0.01$, uncorrected. These results, further suggest that the effect of the two polymorphisms is additive. This is also suggested by the effect size (Cohen's $d$ ) of the difference of signal change in BA 9. The effect size of the difference between COMT Val/Val and Met/Met individuals in BA 9 is $d=1.42$ (Cohen's $d$ ); the effect size between 9/9repeat and 10/10-repeat is $d=0.64$; the effect size between COMT Val/Val DAT 9/10-repeat and COMT Met/Met DAT $10 / 10$-repeat is $d=2.7$.

\section{Discussion}

As predicted, our results indicate that both COMT and DAT genotypes independently predict BOLD signals in the working memory cortical network with COMT Met/Met and DAT 10/ 
10-repeat individuals having a more focused response (lesser activation for similar performance). Moreover, these two genes have an additive effect on this phenotype, because subjects homozygous for COMT Met and DAT 10-repeat alleles have the most focused engagement of the working memory network, whereas subjects homozygous for the COMT Val allele and with DAT 9/10-repeats have the least. These differential responses in the working memory cortical network may result from the differential anatomical and ultrastructural expression of COMT and DAT genes. The COMT Met allele is associated with lower activity of the enzyme, presumably leading to relatively increased synaptic levels of dopamine, which would directly increase the signal-to-noise ratio of pyramidal glutamatergic neurons via stimulation of $D_{1}$ receptors (Seamans and Yang, 2004). The DAT genotype may exert its effect directly in the prefrontal cortex or indirectly via the striatum. In the cortex, DATs tend to be found on nonvaricose axon segments of small diameter, which make symmetric synapses (Sesack et al., 1998; Lewis et al., 2001), with a distribution similar to that of some $\mathrm{D}_{2}$ receptors, a proportion of which are extrasynaptic (Pickel et al., 2002; Negyessy and GoldmanRakic, 2005). Moreover, the functional activity of DAT is regulated by $\mathrm{D}_{2}$ receptors (Meiergerd et al., 1993; Dickinson et al., 1999; Mortensen and Amara, 2003). Thus, the DAT 10-repeat allele associated with increased expression of the gene may presumably lead to relatively decreased extrasynaptic cortical dopamine levels, which would be associated with reduced $\mathrm{D}_{2}$ signaling and with increased GABA release, thus secondarily increasing signal-to-noise ratios of pyramidal neurons (Seamans and Yang, 2004). However, it is also important to consider that the DAT is expressed in low abundance in the cortex, but it is very abundant in the striatum, a key component of the cortico-striato-thalamo-cortical system. It has been argued that it is via this complex, re-entrant system that integrated perceptual, mnemonic, and affective information is synthesized into a coherent stream in working memory (Newman and Grace, 1999). Within this complex circuit, the striatum may serve as a gating system filtering out extraneous inputs and binding together of relevant ones to focus the working memory set. In other words, a primary purpose of striatal circuitry may be to increase the signal-to-noise ratio in the cortex, facilitating binding and suppressing cortical activity not directly contributing to the focus of working memory. Because the net effect of dopamine in the striatum is of increasing activity of thalamo-cortical pathways (Tisch et al., 2004), greater expression of the DAT in the striatum would presumably lead to greater dopamine inactivation with a more focused response of the thalamo-prefrontal pathway. Therefore, it is theoretically possible that our data result from a genetic interaction across the cortico-striatal circuitry, where the effect of COMT would take place in the prefrontal cortex and the effect of DAT either in the cortex or in the striatum, or both. In this case, the interaction in our data would be manifest primarily in the prefrontal cortex, because this is the anatomical region more robustly engaged by our working memory task. Our data do not permit a direct test of these different explanations for the neural mechanism of this additive genetic effect. However, these interpretations are consistent with the known role of dopamine in point-to-point and volume neurotransmission, with the cellular localization of COMT and DAT in the cortex and in the striatum, with the cellular localization of $\mathrm{D}_{1}$ and $\mathrm{D}_{2}$ receptors in the cortex, and with previous functional imaging studies in humans suggesting a critical role for cortical regulation of dopamine via both COMT and DAT (Egan et al., 2001; Mattay et al., 2003).

A limitation of the present study is that we have not measured dopamine directly. Therefore, the effects demonstrated with BOLD fMRI are not necessarily related to dopamine concentrations or release. Instead, they might be related to brain plasticity associated with different molecular mechanisms present at the same time but not necessarily related to these two genotypes. It is also important to note that these genotypes may have an effect on other brain functions. Although the presumed neurobiology of dopamine seems to support a more specific role for modulation of higher cognitive functions, it is also possible that genetic variants of these two genes may exert a more general effect on brain activity, including the default mode network (Raichle et al., 2001; Greicius et al., 2003).

It is also important to consider that we used a simple regression model to account for interacting gene effects. This model assumes that the two genes have a meaningful biological relationship that is linear and continuous. However, there is no evidence supporting a "parametric" relationship between the two genes. Therefore, we performed another regression analysis using a "nonparametric" model for the effect of the two genes. This regression was based on the number of hypothetical "beneficial" alleles (Met and 10-repeat) in each individual, resulting in four groups with 1,2, 3, and 4 of these alleles. Using the same statistical thresholds as in all other statistics, this analysis indicated that the results are very similar (data not shown) to the parametric analysis, suggesting that the effects are not a function of the statistical model used.

To our knowledge, this is the first study in humans of an additive genetic effect of two dopamine regulating genes on the working memory cortical activity phenotype. Elucidation of the genetic factors contributing to individual variation in working memory cortical activity has profound implications for several medical conditions in which brain phenotypes may better elucidate new ways for their assessment and treatment.

\section{References}

Bertolino A, Blasi G, Caforio G, Latorre V, De Candia M, Rubino V, Callicott JH, Mattay VS, Bellomo A, Scarabino T, Weinberger DR, Nardini M (2004a) Functional lateralization of the sensorimotor cortex in patients with schizophrenia: effects of treatment with olanzapine. Biol Psychiatry 56:190-197.

Bertolino A, Caforio G, Blasi G, De Candia M, Latorre V, Petruzzella V, Altamura M, Nappi G, Papa S, Callicott JH, Mattay VS, Bellomo A, Scarabino T, Weinberger DR, Nardini M (2004b) Interaction of COMT Val108/158 Met genotype and olanzapine treatment on prefrontal cortical function in patients with schizophrenia. Am J Psychiatry 161:1798-1805.

Blasi G, Mattay VS, Bertolino A, Elvevag B, Callicott JH, Das S, Kolachana BS, Egan MF, Goldberg TE, Weinberger DR (2005) Effect of catechol-Omethyltransferase val158met genotype on attentional control. J Neurosci 25:5038-5045.

Callicott JH, Mattay VS, Bertolino A, Finn K, Coppola R, Frank JA, Goldberg TE, Weinberger DR (1999) Physiological characteristics of capacity constraints in working memory as revealed by functional MRI. Cereb Cortex 9:20-26.

Callicott JH, Bertolino A, Mattay VS, Langheim FJ, Duyn J, Coppola R, Goldberg TE, Weinberger DR (2000) Physiological dysfunction of the dorsolateral prefrontal cortex in schizophrenia revisited. Cereb Cortex 10:1078-1092.

Chen J, Lipska BK, Halim N, Ma QD, Matsumoto M, Melhem S, Kolachana BS, Hyde TM, Herman MM, Apud J, Egan MF, Kleinman JE, Weinberger DR (2004) Functional analysis of genetic variation in catechol-Omethyltransferase (COMT): effects on mRNA, protein, and enzyme activity in postmortem human brain. Am J Hum Genet 75:807-821. 
Cragg SJ, Rice ME (2004) DAncing past the DAT at a DA synapse. Trends Neurosci 27:270-277.

Dickinson SD, Sabeti J, Larson GA, Giardina K, Rubinstein M, Kelly MA, Grandy DK, Low MJ, Gerhardt GA, Zahniser NR (1999) Dopamine D2 receptor-deficient mice exhibit decreased dopamine transporter function but no changes in dopamine release in dorsal striatum. J Neurochem 72:148-156.

Diop L, Gottberg E, Briere R, Grondin L, Reader TA (1988) Distribution of dopamine D1 receptors in rat cortical areas, neostriatum, olfactory bulb and hippocampus in relation to endogenous dopamine contents. Synapse 2:395-405.

Egan MF, Goldberg TE, Kolachana BS, Callicott JH, Mazzanti CM, Straub RE, Goldman D, Weinberger DR (2001) Effect of COMT Val108/158 Met genotype on frontal lobe function and risk for schizophrenia. Proc Natl Acad Sci USA 98:6917-6922.

Greicius MD, Krasnow B, Reiss AL, Menon V (2003) Functional connectivity in the resting brain: a network analysis of the default mode hypothesis. Proc Natl Acad Sci USA 100:253-258.

Heinz A, Goldman D, Jones DW, Palmour R, Hommer D, Gorey JG, Lee KS, Linnoila M, Weinberger DR (2000) Genotype influences in vivo dopamine transporter availability in human striatum. Neuropsychopharmacology 22:133-139.

Huotari M, Santha M, Lucas LR, Karayiorgou M, Gogos JA, Mannisto PT (2002) Effect of dopamine uptake inhibition on brain catecholamine levels and locomotion in catechol- $O$-methyltransferase-disrupted mice. J Pharmacol Exp Ther 303:1309-1316.

Karoum F, Chrapusta SJ, Egan MF (1994) 3-Methoxytyramine is the major metabolite of released dopamine in the rat frontal cortex: reassessment of the effects of antipsychotics on the dynamics of dopamine release and metabolism in the frontal cortex, nucleus accumbens, and striatum by a simple two pool model. J Neurochem 63:972-979.

Lachman HM, Papolos DF, Saito T, Yu YM, Szumlanski CL, Weinshilboum RM (1996) Human catechol-O-methyltransferase pharmacogenetics: description of a functional polymorphism and its potential application to neuropsychiatric disorders. Pharmacogenetics 6:243-250.

Lewis DA, Melchitzky DS, Sesack SR, Whitehead RE, Auh S, Sampson A (2001) Dopamine transporter immunoreactivity in monkey cerebral cortex: regional, laminar, and ultrastructural localization. J Comp Neurol 432:119-136.

Lotta T, Vidgren J, Tilgmann C, Ulmanen I, Melen K, Julkunen I, Taskinen J (1995) Kinetics of human soluble and membrane-bound catechol $O$ methyltransferase: a revised mechanism and description of the thermolabile variant of the enzyme. Biochemistry 34:4202-4210.

Matsumoto M, Weickert CS, Akil M, Lipska BK, Hyde TM, Herman MM, Kleinman JE, Weinberger DR (2003) Catechol O-methyltransferase mRNA expression in human and rat brain: evidence for a role in cortical neuronal function. Neuroscience 116:127-137.

Mattay VS, Tessitore A, Callicott JH, Bertolino A, Goldberg TE, Chase TN, Hyde TM, Weinberger DR (2002) Dopaminergic modulation of cortical function in patients with Parkinson's disease. Ann Neurol 51:156-164.

Mattay VS, Goldberg TE, Fera F, Hariri AR, Tessitore A, Egan MF, Kolachana
B, Callicott JH, Weinberger DR (2003) Catechol O-methyltransferase val158-met genotype and individual variation in the brain response to amphetamine. Proc Natl Acad Sci USA 100:6186-6191.

Meiergerd SM, Patterson TA, Schenk JO (1993) D2 receptors may modulate the function of the striatal transporter for dopamine: kinetic evidence from studies in vitro and in vivo. J Neurochem 61:764-767.

Mill J, Asherson P, Browes C, D'Souza U, Craig I (2002) Expression of the dopamine transporter gene is regulated by the $3^{\prime}$ UTR VNTR: evidence from brain and lymphocytes using quantitative RT-PCR. Am J Med Genet 114:975-979.

Mortensen OV, Amara SG (2003) Dynamic regulation of the dopamine transporter. Eur J Pharmacol 479:159-170.

Negyessy L, Goldman-Rakic PS (2005) Subcellular localization of the dopamine D2 receptor and coexistence with the calcium-binding protein neuronal calcium sensor-1 in the primate prefrontal cortex. J Comp Neurol 488:464-475.

Newman J, Grace AA (1999) Binding across time: the selective gating of frontal and hippocampal systems modulating working memory and attentional states. Conscious Cogn 8:196-212.

Pickel VM, Garzon M, Mengual E (2002) Electron microscopic immunolabeling of transporters and receptors identifies transmitter-specific functional sites envisioned in Cajal's neuron. Prog Brain Res 136:145-155.

Raichle ME, MacLeod AM, Snyder AZ, Powers WJ, Gusnard DA, Shulman GL (2001) A default mode of brain function. Proc Natl Acad Sci USA 98:676-682.

Seamans JK, Yang CR (2004) The principal features and mechanisms of dopamine modulation in the prefrontal cortex. Prog Neurobiol 74:1-58.

Sesack SR, Hawrylak VA, Matus C, Guido MA, Levey AI (1998) Dopamine axon varicosities in the prelimbic division of the rat prefrontal cortex exhibit sparse immunoreactivity for the dopamine transporter. J Neurosci 18:2697-2708.

Tisch S, Silberstein P, Limousin-Dowsey P, Jahanshahi M (2004) The basal ganglia: anatomy, physiology, and pharmacology. Psychiatr Clin North Am 27:757-799.

Tunbridge EM, Bannerman DM, Sharp T, Harrison PJ (2004) Catechol-Omethyltransferase inhibition improves set-shifting performance and elevates stimulated dopamine release in the rat prefrontal cortex. J Neurosci 24:5331-5335.

Vandenbergh DJ, Persico AM, Hawkins AL, Griffin CA, Li X, Jabs EW, Uhl GR (1992) Human dopamine transporter gene (DAT1) maps to chromosome 5p15.3 and displays a VNTR. Genomics 14:1104-1106.

van Dyck CH, Malison RT, Jacobsen LK, Seibyl JP, Staley JK, Laruelle M, Baldwin RM, Innis RB, Gelernter J (2005) Increased dopamine transporter availability associated with the 9-repeat allele of the SLC6A3 gene. J Nucl Med 46:745-751.

VanNess SH, Owens MJ, Kilts CD (2005) The variable number of tandem repeats element in DAT1 regulates in vitro dopamine transporter density. BMC Genet 6:55.

Zoli M, Torri C, Ferrari R, Jansson A, Zini I, Fuxe K, Agnati LF (1998) The emergence of the volume transmission concept. Brain Res Brain Res Rev 26:136-147. 